[RAdiocarbon, Vol. 19, No. 3, 1977, P. 442-452]

\title{
QUEBEC RADIOCARBON MEASUREMENTS II
}

L BARRETTE, P LASALLE* and C SAMSON

Centre de Recherches Minérales Ministère des Richesses Naturelles

Gouvernement du Québec, Québec

\section{INTRODUCTION}

Experimental procedures and calculation remain almost as previously described in $\mathrm{R}, 1977, \mathrm{v} 19$, p 326-331. The only difference is in the counting equipment. We now use a Beckman LS-230 counter where samples are counted for a total count of about 30,000 .

\section{ACKNOWLEDGMENT}

We wish to thank Richard Morasse who carried out pretreatments, benzene syntheses, and routine operation of the dating equipment.

\section{Richmond Gulf series}

I. GEOLOGIC SAMPLES

\section{A. Quebec, Canada}

These samples were coll 1972 and subm by D Lagarec (except QU-140 coll by H Samson).

\section{QU-140. Richmond I}

$4960 \pm 120$

Wood and peat from contact between silt and peat on a palsa $\mathbf{E}$ of Richmond gulf $\left(56^{\circ} 08^{\prime}, \mathrm{N}, 75^{\circ} 55^{\prime} \mathrm{W}\right)$, +132m. Comment (DL): date is minimum for emergence since a lagoon occupied site after marine stage and since time must also be allowed for peat growth.

QU-141. Richmond II

$1950 \pm 90$ $+12 \mathrm{~m}$.

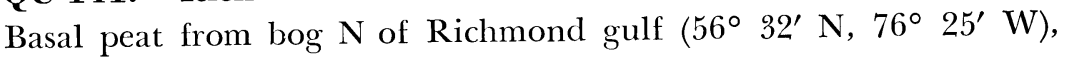

QU-142. Richmond III

$4720 \pm 120$

Roots from contact between silt and peat in a palsa $S$ of Richmond gulf $\left(56^{\circ} 7^{\prime} \mathrm{N}, 76^{\circ} 28^{\prime} \mathrm{W}\right),+84 \mathrm{~m}$.

\section{QU-143. Nastapoka River}

$7760 \pm 130$

Basal peat of a palsa near mouth of Nastapoka R $\left(56^{\circ} 48^{\prime} \mathrm{N}, 76^{\circ} 13^{\prime}\right.$ $\mathrm{W}),+186 \mathrm{~m}$.

\section{QU-144. Boniface River}

Wood from base of $255 \mathrm{~cm}$ peat layer of palsa near Boniface $\mathrm{R}\left(57^{\circ}\right.$ $\left.47^{\prime} \mathrm{N}, 76^{\circ} 17^{\prime} \mathrm{W}\right),+135 \mathrm{~m}$.

General Comment (DL): from above dates we can expect that deglaciation is much older than $7500 \mathrm{BP}$ as proposed by Prest (1970). On the other hand, taking into account time differences between site emergence and

* Exploration Géologique, Ministère des Richesses naturelles. 
peat growth and differences between dates from shells and organic sam$\mathrm{N}$ of Poste-de-la-Baleine.

QU-145. Mont Jacques-Cartier

$1420 \pm 150$

Wood underlying a non-sorted stripe at lower limit of occurrence of phenomenon on Mont Jacques-Cartier $\left(49^{\circ} 00^{\prime} \mathrm{N}, 65^{\circ} 57^{\prime} \mathrm{W}\right),+1110 \mathrm{~m}$. Coll 1973 by F Boudreau and subm by DL. Comment (DL): minimum for non-sorted stripe period of activity in that area.

\section{Lake Mimi series}

QU-54. Lake Mimi I

$7700 \pm 310$ 400 to $410 \mathrm{~cm}$

QU-70. Lake Mimi II

$9460 \pm 280$

425 to $435 \mathrm{~cm}$

QU-56. Lake Mimi III

455 to $460 \mathrm{~cm}$

$10,180 \pm 330$

QU-67. Lake Mimi IV

470 to $480 \mathrm{~cm}$

$9770 \pm 260$

\section{QU-55. Lake Mimi V}

$500-515 \mathrm{~cm}$

$11,050 \pm 460$

Five samples of gyttja coll by P Poulin with a Livingstone sampler in 1971, from site ca $4 \mathrm{~km} \mathrm{NW}$ of village of Les Eboulements, Quebec $\left(47^{\circ}\right.$ $29^{\prime} 50^{\prime \prime} \mathrm{N}, 70^{\circ} 22^{\prime} 35^{\prime \prime} \mathrm{W}$ ). Comment (PP): QU-55 give a minimum age for ice retreat in Mont des Eboulements area. QU-56 dates inversion of APNAP curve in pollen diagram (Richard \& Poulin, 1976) and suggests slight climatic deterioration for the time. The 3 other dates help confirm the others, and also help to estimate rate of sedimentation for that lake.

\section{Baie-des-Sables/Trois-Pistoles series}

Samples of present series coll and subm by J Locat, Baie-des-Sables/ Trois-Pistoles region (Locat, 1976).

\section{QU-261. Price}

$$
11,100 \pm 370
$$

Shells (Mytilus edulis dominant but assoc with Macoma balthica, Hiatella arctica, Mytilu edulis, Balanus sp Mya arenaria) coll in gravelly sand layer. Site $\left(48^{\circ} 36^{\prime} 38^{\prime \prime} \mathrm{N}, 68^{\circ} 06^{\prime} 20^{\prime \prime} \mathrm{W}\right)$, +62m, in littoral sands and gravels below Price delta $(+76 \mathrm{~m})$. Sea level assoc with these fossils was between 62 and $76 \mathrm{~m}$ above present sea level. Shorelines at this site are very well-defined, and disappear at $+70 \mathrm{~m}$. Comment $(\mathrm{JL})$ : date is maximum for marine water level assoc with position of present $+62 \mathrm{~m}$ contour
line. 
QU-262. Mont-Joli

$11,380 \pm 410$

Shells (Mytilus edulis o Balanus sp) coll in coarse sand overlain by oxidized fine sand. At this site $\left(48^{\circ} 35^{\prime} 35^{\prime \prime} \mathrm{N}, 68^{\circ} 12^{\prime} 32^{\prime \prime} \mathrm{W}\right),+75 \mathrm{~m}$, $1.5 \mathrm{~km} \mathrm{~W}$ of Mont-Joli, littoral sediments form a terrace in which sec was cut. Comment (JL): result dates maximum of sea level at $+75 \mathrm{~m}$.

\section{QU-263. St-Anaclet}

$12,220 \pm 450$

Shels (Mya sp separated from several species) from fresh ditch in clayey sand overlain by thin littoral fine to medium sand, W of St-Anaclet $\left(48^{\circ} 29^{\prime} 00^{\prime \prime} \mathrm{N}, 68^{\circ} 22^{\prime} 40^{\prime \prime} \mathrm{W}\right),+82 \mathrm{~m}$. Sediments may be closely related to St-Anaclet delta $(+125 \mathrm{~m})$ to the $\mathrm{S}$. Comment $(\mathrm{JL})$ : age is maximum for position of sea level at $+82 \mathrm{~m}$.

QU-264. St-Donat

$13,360 \pm 320$

Shells (Hiatella arctica, Mya truncata \& Balanus hameri), bivalves well-preserved, from marine clay grading upward into lacustrine varved sediments ( 48 couplets), in turn overlain by fluvial sand and gravel $\left(48^{\circ}\right.$ $\left.30^{\prime} 11^{\prime \prime} \mathrm{N}, 68^{\circ} 15^{\prime} 55^{\prime \prime} \mathrm{W}\right)$, ca $+90 \mathrm{~m}$. Comment $(\mathrm{JL})$ : result is close to time of deglaciation, ca 13,500 BP, as suggested by Elson (1969) for Rivière-duLoup/Trois-Pistoles area. It also dates time of maximum marine inundation in Luceville/St-Donat area and must be related to Luceville delta at $+126 \mathrm{~m}$.

\section{QU-265. Baie-des-Sables}

$2240 \pm 140$

Shells (Mesodesma arctica) from littoral sediments made of gravelly sand near village of Baie-des-Sables on Ste-Flavie terrace $\left(48^{\circ} 43^{\prime} 37^{\prime \prime} \mathrm{N}\right.$, $\left.67^{\circ} 53^{\prime} 00^{\prime \prime} \mathrm{W}\right),+4 \mathrm{~m}$. Species encountered has never been found in higher deposit. Only unbroken valves (ca 50\% were used. Comment (JL): result dates marine level $(+4 \mathrm{~m})$ coinciding with terrace surface.

\section{QU-266. Luceville}

$10,400 \pm 320$

Shells (Mytilus edulis) from gravel pit in littoral sediments at ca $3 \mathrm{~km}$ SW of Luceville $\left(48^{\circ} 30^{\prime} 21^{\prime \prime} \mathrm{N}, 68^{\circ} 21^{\prime} 30^{\prime \prime} \mathrm{W}\right)$, $+70 \mathrm{~m}$. Gravel pit extends ca $50 \mathrm{~m}$ into marine terrace lying at ca $+73 \mathrm{~m}$ in area. Comment $(\mathrm{JL})$ : result dates marine level assoc with $+73 \mathrm{~m}$ terrace.

\section{QU-267. Grand Métis}

$11,590 \pm 430$

Shells (Macoma balthica, unbroken valves, and gastropods and Mya $\mathrm{sp}$ ) coll near Grand Métis, ca $10 \mathrm{~km} \mathrm{E}$ of Mont-Joli, on Bic terrace, near SE edge of old channel $\left(48^{\circ} 36^{\prime} 47^{\prime \prime} \mathrm{N}, 68^{\circ} 06^{\prime} 00^{\prime \prime} \mathrm{W}\right)$, $+30 \mathrm{~m}$. Comment $(\mathrm{JL})$ : date seems too old; possible reworking of higher and older deposits suggested by location of site within erosion channel. Main sea level assoc with this site is ca $+53 \mathrm{~m}$, corresponding to adjacent shorelines. Shells may also have been contaminated by humic acid or carbonate fertilizers.

QU-268. St-Octave

$11,360 \pm 290$

Shells (Mytilus edulis) from medium sand to medium gravel near village of St-Octave-de-Métis $\left(48^{\circ} 36^{\prime} 48^{\prime \prime} \mathrm{N}, 68^{\circ} 06^{\prime} 22^{\prime \prime} \mathrm{W}\right)$. Top of sec 
ca $2.4 \mathrm{~m}$ above fossiliferous layer and is crest of a emerged beach deposit. Comment (JL): result dates marine level assoc with present position of $+62 \mathrm{~m}$ contour line.

\section{QU-271. St-Fabien I \\ QU-272. St-Fabien II}

$$
\begin{aligned}
& 13,390 \pm 690 \\
& 12,300 \pm 260
\end{aligned}
$$

Shells (Hiatella arctica for QU-271 assoc with Balanus hameri, Macoma balthica and Mya arenaria for QU-272) coll in fossiliferous till inclusion incorporated into ice-contact sediments in St-Fabien delta near St-Fabien $\left(48^{\circ} 18^{\prime} 25^{\prime \prime} \mathrm{N}, 68^{\circ} 51^{\prime} 12^{\prime \prime} \mathrm{W}\right)$, +138m. Delta reaches maximum alt of $155 \mathrm{~m}$ which corresponds to maximum sea level. This very interesting site is highest Quaternary marine macrofossil locality in Gaspe. Dates come from shells coll from 2 different inclusions; QU-271 is more reliable. Comment $(\mathrm{JL})$ : results date marine level assoc with position of present
$+138 \mathrm{~m}$ contour line.

\section{James Bay series}

All samples in this group were coll 1973-5 by L Hardy.

QU-248. Fort George I

$4470 \pm 170$

Shells (Hiatella arctica and probably Clinocardium ciliatum) in sandy silt, 16km E of Fort George $\left(53^{\circ} 45^{\prime} 30^{\prime \prime} \mathrm{N}, 78^{\circ} 52^{\prime} 30^{\prime \prime} \mathrm{W}\right),+16 \mathrm{~m}$.

QU-121. Fort George II

$4110 \pm 120$

Shells (Hiatella arctica) in beach gravel $22 \mathrm{~km}$ E of Fort George $\left(53^{\circ}\right.$ $\left.44^{\prime} 25^{\prime \prime} \mathrm{N}, 78^{\circ} 45^{\prime} 10^{\prime \prime} \mathrm{W}\right)$. Comment $(\mathrm{LH})$ : dates position of Tyrrell sea
at present $+40 \mathrm{~m}$ contour line.

\section{QU-256. Fort George III}

$$
5080 \pm 180
$$

Shells (Hiatella arctica) in coarse beach gravel $50 \mathrm{~km}$ E of Fort George $\left(53^{\circ} 40^{\prime} \mathrm{N}, 78^{\circ} 20^{\prime} \mathrm{W}\right),+99 \mathrm{~m}$. Comment $(\mathrm{LH})$ : dates position of Tyrrell sea at present $99 \mathrm{~m}$ contour line.

\section{QU-119. Fort George IV}

Shells (Hiatella arctica and debris of probable Pecten sp) in shore deposits of Tyrrell sea $65 \mathrm{~km} \mathrm{E}$ of Fort George $\left(53^{\circ} 43^{\prime} \mathrm{N}, 77^{\circ} 58^{\prime} \mathrm{W}\right)$, $+172 \mathrm{~m}$. Comment $(\mathrm{LH})$ : dates position of Tyrrell seat at present $172 \mathrm{~m}$
contour line.

\section{QU-247. Fort George V}

$6910 \pm 350$

Shells (Hiatella arctica) in beach gravel, $85 \mathrm{~km} \mathrm{E}$ of Fort George $\left(53^{\circ}\right.$ $\left.44^{\prime} \mathrm{N}, 77^{\circ} 52^{\prime} \mathrm{W}\right),+183 \mathrm{~m}$. Comment $(\mathrm{LH})$ : dates position of Tyrrell sea
at present $183 \mathrm{~m}$ contour line.

\section{QU-245. Fort George VI}

Shells (Hiatella arctica) in nearshore sandy silt deposit $85 \mathrm{~km} \mathrm{E}$ of Fort George $\left(53^{\circ} 42^{\prime} \mathrm{N}, 77^{\circ} 52^{\prime} \mathrm{W}\right)$, +166m. Comment ( $\left.\mathrm{LH}\right)$ : maximum age for retreat of water plane of Tyrrell Sea at position of present $166 \mathrm{~m}$ 
contour line. From field relations, it dates approx position of Tyrrell sea at present $185 \mathrm{~m}$ contour line.

QU-246. Mattagami-La Grande Road, Mile $363 \quad \mathbf{7 2 2 0} \pm 330$

Shells (Hiatella arctica) coll in reworked fluvio-glacial gravelly sand, $\left(53^{\circ} 34^{\prime} 40^{\prime \prime} \mathrm{N}, 77^{\circ} 40^{\prime} \mathrm{W}\right)$, +171m. Comment (LH): minimum age for deglaciation and arrival of sea at this point.

QU-249. Mattagami-La Grande Road, Mile $356 \quad 6660 \pm 190$

Shells (Hiatella arctica) in nearshore deposit of Tyrrell sea $\left(53^{\circ} 28^{\prime} \mathrm{N}\right.$, $\left.77^{\circ} 30^{\prime} \mathrm{W}\right),+164 \mathrm{~m}$. Comment $(\mathrm{LH})$ : from field relations dates position of Tyrrell sea at present $175 \mathrm{~m}$ contour line.

Mattagami-La Grande Road, Mile 344

QU-122.

$7880 \pm 160$

(Chlamys islandicus), $+162 \mathrm{~m}$

QU-124.

$7750 \pm 180$

(Hiatella arctica and other broken shells), $+162.1 \mathrm{~m}$

Shells from 2 different levels in glacio-marine sandy silt underlying beach gravel and overlying Sakami moraine material, $4.5 \mathrm{~km} \mathrm{~W}$ of Mile Post $344\left(53^{\circ} 21^{\prime} \mathrm{N}, 77^{\circ} 34^{\prime} \mathrm{W}\right)$. Comment $(\mathrm{LH})$ : these 2 dates should give approx time of marine invasion in James bay lowlands.

QU-250. Mattagami-La Grande Road, Mile $292 \quad 6930 \pm 190$

Shells (Hiatella arctica and probably Chlamys islandicus) in nearshore sandy and pebbly silt $\left(52^{\circ} 46^{\prime} 30^{\prime \prime} \mathrm{N}, 77^{\circ} 18^{\prime} 00^{\prime \prime} \mathrm{W}\right)$, +195m. Comment $(\mathrm{LH})$ : dates position of Tyrrell sea at present $195 \mathrm{~m}$ contour line.

QU-253. Mattagami-La Grande Road, Mile $275 \quad 6950 \pm 210$

Shells (Hiatella arctica and Mya truncata) in beach sand $\left(52^{\circ} 34^{\prime} \mathrm{N}\right.$, $\left.77^{\circ} 20^{\prime} \mathrm{W}\right),+200 \mathrm{~m}$. Comment $(\mathrm{LH})$ : dates position of Tyrrell sea at present $200 \mathrm{~m}$ contour line.

QU-258. Mattagami-La Grande Road, Mile $263 \quad \mathbf{7 4 4 0} \pm 210$

Shells (Hiatella arctica) in nearshore sandy silt $\left(52^{\circ} 25^{\prime} \mathrm{N}, 77^{\circ} 55^{\prime} \mathrm{W}\right)$, $+200 \mathrm{~m}$. Comment (LH): from field relations, dates position of Tyrrell sea at present $215 \mathrm{~m}$ contour line.

QU-368. Eastmain River

$\mathbf{7 4 4 0} \pm 180$

Shells in silty clay at base of marine (Tyrrell sea formation) $\mathrm{N}$ bank of Eastmain R, $7.3 \mathrm{~km}$ upstream from its junction with Opinaca $\mathrm{R}\left(52^{\circ} 13^{\prime}\right.$ $\left.20^{\prime \prime} \mathrm{N}, 77^{\circ} 55^{\prime} \mathrm{W}\right),+9 \mathrm{~m}$. Comment $(\mathrm{LH})$ : dates approx time of marine invasion (Tyrrell sea in James bay lowlands). Also a minimum age for deglaciation at this point.

QU-254. Eastmain River

$7140 \pm 210$

Shells (Hiatella arctica) in beach gravel. $1 \mathrm{~km} \mathrm{~S}$ of Eastmain $\mathrm{R}$ along Matagami-La Grande $\mathrm{Rd}\left(52^{\circ} 18^{\prime} 30^{\prime \prime} \mathrm{N}, 77^{\circ} 05^{\prime} \mathrm{W}\right)$, +218m. Comment (LH): dates position of Tyrell sea at present $225 \mathrm{~m}$ contour line. 
QU-252. Mattagami-La Grande Road, Mile $236 \quad 7030 \pm 210$

Shells (Hiatella arctica) in nearshore sandy silt $\left(52^{\circ} 13^{\prime} \mathrm{N}, 77^{\circ} 08^{\prime} \mathrm{W}\right)$, $+222 \mathrm{~m}$. Comment $(\mathrm{LH})$ : dates position of Tyrrell sea at present $230 \mathrm{~m}$
contour line.

\section{B. Spitzbergen, Svalbard}

\section{West Spitzbergen series}

These 2 samples were coll 1973 and subm by J Fabiszewski and $\mathrm{K}$ Pekala, Polish Spitsbergen Expedition.

\section{QU-156. Ralstranda bog}

Basal part of peat bog, $1.7 \mathrm{~m}$ under surface, containing remains of tundra plants, accumulated on 3rd marine terrace at Ralstranda $\left(77^{\circ} 0^{\prime}\right.$ $\left.50^{\prime \prime} \mathrm{N}, 15^{\circ} 19^{\prime} 55^{\prime \prime} \mathrm{E}\right), \mathrm{ca}+25 \mathrm{~m}$. Comment (JF): result indicates fast peat accumulation in polar conditions and dates older Salix tundra, existing during previous glacier readvance.

\section{QU-157. Eimfiellet nunatak}

Organic layer under $60 \mathrm{~cm}$ of Werenskioldbreen nunaterial around Glacier Comment (JF): Comment (JF): age of tundra growing on nunatak before deposition of morainic material; also maximum age for recent glacial activity in area.

$$
\text { II. SOIL SAMPLES }
$$

\section{A. Quebec, Canada}

All soil samples were pretreated by collectors and subm as strontium carbonate. Ages when present are only apparent ages. ${ }^{14} \mathrm{C}$ concentrations are given as \% modern relative to 0.95 NBS oxalic acid.

\section{Horizon A series}

Samples coll 1974 and subm by Y A Martel and P LaSalle. Bulk samples were taken from 0 to $18 \mathrm{~cm}$ topsoil, Ap horizon, of a cultivated clay loam soil classified as Kamouraska series and in Gleysolic order ( $47^{\circ}$ $\left.20^{\prime} \mathrm{N}, 70^{\circ} 02^{\prime} \mathrm{W}\right)$. Comment (YAM): studied to determine stability of soil organic matter in cultivated topsoil.

\section{QU-131. Kamouraska, topsoil}

Total soil organic matter. Roots were removed by water flotation and carbonates by IN HCl treatment.

QU-129. Kamouraska, humic acids

$$
1220 \pm 150
$$

$86 \pm 2 \%$ modern

Fraction of QU-131 extracted with $0.5 \mathrm{~N} \mathrm{NaOH}$ and precipitated at pH 2 with $1 \mathrm{~N} \mathrm{HCl.}$

QU-130. Kamouraska, Humin I

Fraction of QU-131 unextractable with $0.5 \mathrm{~N} \mathrm{NaOH}$. 


\section{QU-185. Kamouraska, Humin II} $\mathrm{NaOH}$.

Fraction of topsoil, different from QU-131, unextractable with $0.5 \mathrm{~N}$

QU-132. Kamouraska, nonhydrolizable

$$
\begin{array}{r}
1530 \pm 110 \\
83 \pm 1 \% \text { modern }
\end{array}
$$

Total soil was hydrolyzed using $0.5 \mathrm{~N}$ and $6 \mathrm{~N}$ HCl (Martel Paul, 1974). Nonhydrolyzable residue is dated.

\section{Horizon C series}

Soil samples, coll 1975 and subm by YAM and PL, were taken below root zone and total soil organic matter was dated after carbonates were removed with $1 \mathrm{~N} \mathrm{HCl}$. Comment (YAM): studied to determine utility of such dates in estimating age of soil formation in zone of Champlain sea. QU-310 is shell sample related to corresponding soil.
QU-188. De L'Anse
$1850 \pm 90$
$79 \pm 1 \%$ modern

Soil coll at 210 to $240 \mathrm{~cm}$ below surface, on De L'Anse soil series, Micmac terrace, at La Pocatière $\left(47^{\circ} 22^{\prime} \mathrm{N}, 70^{\circ} 03^{\prime} \mathrm{W}\right)$, $+6 \mathrm{~m}$.
QU-232. Kamouraska
$11,730 \pm 310$
$23 \pm 1 \%$ modern

Soil coll at 240 to $270 \mathrm{~cm}$ below surface, on Kamouraska soil series at La Pocatière $\left(47^{\circ} 20^{\prime} \mathrm{N}, 70^{\circ} 02^{\prime} \mathrm{W}\right),+115 \mathrm{~m}$.

\section{QU-312. Fouquette}

$$
\begin{array}{r}
10,180 \pm 270 \\
28 \pm 1 \% \text { modern }
\end{array}
$$

Soil coll at 150 to $180 \mathrm{~cm}$ below surface on Fouquette soil series at St-Hélène de Kamouraska $\left(47^{\circ} 35^{\prime} \mathrm{N}, 69^{\circ} 45^{\prime} \mathrm{W}\right)$, $+140 \mathrm{~cm}$.

\section{QU-313. Ste-Rosalie}

$$
16,170 \pm 230
$$

$13 \pm 1 \%$ modern

Soil col at 300 to $330 \mathrm{~cm}$ below surface on Ste-Rosalie soil series at L'Acadie $\left(45^{\circ} 19^{\prime} \mathrm{N}, 73^{\circ} 16^{\prime} \mathrm{W}\right)$, + $150 \mathrm{~m}$.

\section{QU-311. St-Rédempteur}

$12,340 \pm 340$ $22 \pm 1 \%$ modern

Soil coll at $260 \mathrm{~cm}$ below surface at St-Rédempteur $\left(46^{\circ} 43^{\prime} \mathrm{N}, 71^{\circ} 17^{\prime}\right.$ $\mathrm{W}),+60 \mathrm{~m}$.

QU-310. St-Rédempteur, shells

$$
\begin{array}{r}
9210 \pm 130 \\
32 \pm 1 \% \text { modern }
\end{array}
$$

Shells coll at same location and depth as QU-311.

$$
\text { III. ARCHAEOLOGIC SAMPLES }
$$

All archaeologic samples were coll within prov of Quebec and subm by people from Provincial Cultural Affairs Dept. 
Rivière-au-Bouleau series

A. Quebec, Canada

Samples from Rivière-au-Bouleau, Quebec (50 $\left.17^{\prime} \mathrm{N}, 65^{\circ} 30^{\prime} \mathrm{W}\right)$. Coll and subm by D Chevrier.
QU-114. Rivière-au-Bouleau I
$1770 \pm 110$
QU-115. Rivière-au-Bouleau II
$2870 \pm 180$
QU-116. Rivière-au-Bouleau III
$3220 \pm 240$

Charcoal assigned to Middle Woodland because of archaeol assemblage. Two periods of occupation postulated, not separated by a long time. Comment (DC): QU-114 seems post probable date; other 2 dates are too old. Last 2 samples very small. Benzene obtained was minimal.

QU-234. Rivière-au-Bouleau IV

$1280 \pm 170$

Charcoal from site which should have had 2 periods of occupation; opinion based on pedol, geomorphol, and bot reasons. Comment (DC): dates 1st occupation.

QU-236. Rivière-au-Bouleau V

$1620 \pm 340$

Charcoal from site which should have been occupied before $5000 \mathrm{BP}$. Comment (DC): date too young. Probable contamination by rootlets or humic acids.

QU-237. Rivière-au-Bouleau VI

$310 \pm 70$

Charcoal from site which should have been occupied between 1000 and $1500 \mathrm{BP}$. Comment (DC): sample was not directly linked to archaeol levels; date tends to confirm that sample does not belong to prehistoric
occupation.

\section{QU-238. Rivière-au-Bouleau VII}

$1140 \pm 210$

Charcoal from site which should have been occupied certainly before 6000 BP. Comment (DC): date too young, probably contaminated by rootlets or humic acids.

\section{QU-117. Chambly I \\ QU-118. Chambly II}

$$
1130 \pm 90
$$

Charcoal from a site $\left(45^{\circ} 23^{\prime} 36^{\prime \prime} \mathrm{N}, 73^{\circ} 15^{\prime} 90^{\prime \prime} \mathrm{1160} \pm \mathbf{1 1 0}\right.$ should have been occupied uring Archaic period. Coll by $G$ Frenette and N Clermont. Comment (NC): dates should be 1500 yr older. Small rootlets present in zone of coll together with fertilization of topsoil could have contaminated samples.

\section{QU-235. Lotbinière}

$$
1630 \pm 310
$$

Charcoal from a site $\left(46^{\circ} 31^{\prime} 00^{\prime \prime} \mathrm{N}, 71^{\circ} 53^{\prime} 30^{\prime \prime} \mathrm{W}\right)$ which seems to correspond to same assemblage of artifacts as those of Owasco culture, New York (Ritchie, 1965) coll by R Ribes. Comment (RR): date does not 
agree with opinion expressed above, but seems to agree with that obtained on charcoal at a site near Trois-Rivières, Bourassa site, which corresponds to Middle Woodland.

\section{St-André-de-Kamouraska series}

Samples coll by P Dumais near St-André.

QU-357. Rivière-des-Caps

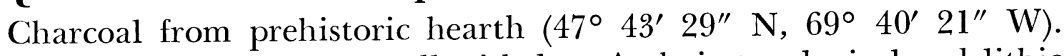
Comment (PD): date agrees well with late Archaic typological and lithic assemblage.

\section{QU-358. St-André I}

$630 \pm 80$

Fragmented charcoal from test pits $\left(47^{\circ} 40^{\prime} 22^{\prime \prime} \mathrm{N}, 69^{\circ} 44^{\prime} 38^{\prime \prime} \mathrm{W}\right)$. Comment (PD): site considered to be from Archaic period so date appears too young. Sample may have been contaminated by later human activities at site.

QU-359. St-André II $370 \pm 90$

Fragmented charcoal from test pits $\left(47^{\circ} 43^{\prime} 26^{\prime \prime} \mathrm{N}, 69^{\circ} 40^{\prime} 36^{\prime \prime} \mathrm{W}\right)$. Comment (PD): date seems too young as material recovered bore no sign of European occupation. Sample may have been contaminated and not even related to archaeol site.

\section{Lanoraie series}

Samples coll by G Barré.

\section{QU-218. Lanoraie I}

$660 \pm 100$

QU-220. Lanoraie III

$810 \pm 160$

Wood and charcoal from a site $\left(45^{\circ} 57^{\prime} \mathrm{N}, 73^{\circ} 13^{\prime} \mathrm{W}\right)$ whose occupation has been assigned to early stage of Iroquois prehistory. Comment (GB): dates confirm interpretation given above.

\section{QU-219. Lanoraie II}

$1610 \pm 160$

\section{QU-221. Lanoraie IV}

$1790 \pm 280$

Wood and charcoal from pit and post mold in Iroquois long house $\left(45^{\circ} 59^{\prime} 15^{\prime \prime} \mathrm{N}, 73^{\circ} 8^{\prime} 50^{\prime \prime} \mathrm{W}\right)$. Comment (GB): based on artifacts and other features found at site, dates do not seem to correspond with occupation of sites which should have been somewhere in $14^{\text {th }}$ century.

\section{Tracy series}

Samples coll by J Mandeville near Tracy (45 $59^{\prime} 5^{\prime \prime} \mathrm{N}, 73^{\circ} 8^{\prime} 50^{\prime \prime} \mathrm{W}$ ) and subm by $\mathrm{G}$ Barré. 
QU-222. Tracy I

$410 \pm 120$

Charcoal from carbonized habitation post assoc with Iroquois artifacts dated archaeol ca $1500 \mathrm{AD}$. Comment (GB): date confirms interpretation given above.

QU.223. Tracy II

$710 \pm 130$

Charcoal from carbonized piece of wood separating 2 skeletons in Iroquois burial. Comment (GB): date, which should be ca $1500 \mathrm{AD}$, appears slightly too old.

\section{N Gaspe Peninsula series}

Samples coll 1974 by J Benmouyal from 3 archaeol sites on $\mathrm{N}$ shore of Gaspe peninsula.

\section{QU-347. Ste-Anne}

$5960 \pm 100$

Charcoal assoc with probable hearth. Habitation site $\left(49^{\circ} 07^{\prime} 35^{\prime \prime} \mathrm{N}\right.$, $66^{\circ} 27^{\prime} 32^{\prime \prime} \mathrm{W}$ ) on a $+45 \mathrm{~m}$ terrace which has given remains of late PaleoIndian tradition. Comment (JB): age estimate, one of earliest dates for a prehistoric site, in Quebec, seems acceptable, although an older one would not have been surprising.
QU-373. Cap-au-Renard, B-10
$5270 \pm 90$
QU-227. Cap-au-Renard, B-1
$4940 \pm 170$
QU-228. Cap-au-Renard, B-2
$4170 \pm 150$
QU-229. Cap-au-Renard, B-4
$3750 \pm 180$
QU-372. Cap-au-Renard, B-7
$2500 \pm 80$

Five charcoal samples, thought to belong to local "Middle-Archaic" tradition, coll on a $+25 \mathrm{~m}$ terrace $\left(49^{\circ} 11^{\prime} 51^{\prime \prime} \mathrm{N}, 66^{\circ} 13^{\prime} 16^{\prime \prime} \mathrm{W}\right)$. This living site has yielded lithic remains in podzol, in $\mathrm{A}_{2}$ and $\mathrm{B}$ horizons but mainly underlying humic level, overlying leached $\left(\mathrm{A}_{2}\right)$ soil. Comment (JB): results range rather widely in time; problem cannot be solved by assoc material. Some samples may represent forest fires, or may be contaminated. However, 2 samples, QU-228 and -229 are assoc with hearth; they were deeply buried in $A_{2}$ horizon, 27 to $32 \mathrm{~cm}$ below surface. They probably best date human occupation.

QU-226. Cap-au-Renard, A-12

$$
\begin{aligned}
& 3030 \pm 470 \\
& 2280 \pm 230 \\
& 1880 \pm 110
\end{aligned}
$$

QU-225. Cap-au-Renard, A-6

QU-374. Cap-au-Renard, A-5/7

QU-224. Cap-au-Renard, A-1 coll on small, badly drained terrace at $+18 \mathrm{~m}$, underlying QU-373 group one. Material remains were under dark humic layer, in dark sand rich in decaying organic matters. Comment (JB): 2 samples, QU-225 and -374, drawn from hearth with fire-cracked rocks, faunal, and other cultural remains, must be favored. 


\section{REFERENCES}

Elson, J A, 1969, Late Quaternary submergence of Quebec: Geog Montreal Rev, v 23, p 247-258.

Locat, J, 1976, Quaternary geology of the Baie-des-Sables/Trois-Pistoles area, Québec; with some emphasis on the Goldthwait sea clays: M Sc thesis, Waterloo Univ, unpub, 214p.

Martel, Y A and Paul, E A, 1974, Effects of cultivation on the organic matter of grassland soils as determined by fractionation and radiocarbon dating: Canadian Jour Soil Sci, v 54, p 419-426.

Prest, V K, 1970, Quaternary geology of Canada, in: Geology and economic minerals of Canada, R J V Douglas (ed), p 677-764.

Richard, P and Poulin, P, 1976, Un diagramme pollinique au Mont des Eboulements, région de Charlevoix, Québec: Canadian Jour Earth Sci, v 13, 6 145-156.

Ritchie, W A, 1965, The Archeology of New York State: New York, Nat Hist Press, $355 \mathrm{p}$.

Walcott, R I, 1972, Late Quaternary vertical movements in eastern North America; quantitative evidence of glacio-isostatic rebound: Geophys \& Space phys rev, v 10, p 849-884. 